01

\title{
Возбуждение и регистрация нелинейного резонанса колебаний пружинного маятника с использованием электромагнитной индукции
}

\author{
(C) И.С. Зейликович, А.В. Никитин, А.Е. Василевич \\ Гродненский государственный университет им. Янки Купалы, \\ 230023 Гродно, Беларусь \\ e-mail: zeylikov@gmail.com
}

Поступило в Редакцию 13 февраля 2019 г.

В окончательной редакции 12 апреля 2019 г.

Принято к публикации 17 июня 2019 г.

Экспериментально и теоретически исследованы нелинейные колебания и резонансы пружинного маятника. Предложен унифицированный посредством электромагнитной индукции способ возбуждения, диссипации и регистрации нелинейных колебаний. Экспериментальные результаты демонстрируют типичные нелинейные эффекты, такие как неизохронность и бистабильность колебаний. Предложена теоретическая модель колебательной системы, приводящая к уравнению Дуффинга, приведены аналитическое решение и результаты численного моделирования, хорошо согласующиеся с экспериментом.

Ключевые слова: нелинейные колебания, резонанс, электромагнитная индукция.

DOI: $10.21883 /$ JTF.2020.01.48653.48-19

\section{Введение}

Нелинейные процессы в колебательных системах играют важную роль не только в механических и электрических процессах, но и определяют физические свойства тел. Изучению нелинейных эффектов в механических и электрических системах уделяется большое внимание [1-3]. Математически чаще всего это изучение базируется на анализе решений (как аналитических, так и численных) уравнений нелинейных колебаний, например, уравнения Дуффинга. Аппаратная реализация установок обеспечивает создание нелинейности колебаний и регистрацию параметров. В работе [3] рассматривается установка для получения нелинейных колебаний математического маятника, в которой нет возможности регулирования коэффициента затухания колебаний. В отечественной литературе нелинейные колебания также изучаются на примере математического маятника [4] или с использованием электронных схем. В то же время опускаются ,детали“ экспериментальной реализации возбуждения и регистрации нелинейных параметров колебаний системы.

В настоящей работе представлена реализация экспериментальной установки для исследования нелинейных колебаний пружинного маятника. Экспериментально и теоретически исследуются нелинейные колебания и резонансы пружинного маятника. Возбуждение колебаний, имитация диссипативных сил, создание нелинейных эффектов и их регистрация реализуются унифицированным способом - только за счет электромагнитной индукции. Предложена теоретическая модель (аналитическая и численная), а также сопоставляются данные, полученные теоретическими и экспериментальными методами.

\section{1. Экспериментальная установка}

На рис. 1 показана схема колебаний пружинного маятника, состоящего из двух одинаковых пружин 1 и цилиндрического магнита 2. Магнит может колебаться вдоль вертикальной оси $x$, проходящей через центры полых катушек 3,5 и 7. Центральный виток катушки 3 совмещен с центром магнита 2 в положении равновесия, центральные витки катушек 5 и 7 смещены относительно центра магнита. Катушка 3 подключена к источнику постоянного напряжения 4, а катушка 5 к источнику переменного напряжения 6. Катушка 7, выходные клеммы которой замкнуты с помощью резистора, соединена с цифровой лабораторией 8 , регистрирующей ЭДС катушки в режиме осциллографа.

\section{2. Теоретическая модель}

Если пренебречь массой пружин и рассматривать малые колебания, то уравнение движения этого маятника можно записать в виде

$$
M \ddot{x}=-\left(k_{1}+k_{2}\right) x-k_{v} \dot{x}+F_{M}+F_{\sim},
$$

где $x$ - смещение груза относительно положения равновесия $[\mathrm{m}] ; M-$ масса груза $[\mathrm{kg}] ; k_{1}, k_{2}$ - коэффициенты жесткости пружин $[\mathrm{N} / \mathrm{m}] ; k_{v}-$ коэффициент сопротивления (диссипации) $[\mathrm{N} \cdot \mathrm{s} / \mathrm{m}] ; F_{\mathrm{M}}-$ сила, обеспечивающая нелинейность колебаний $[\mathrm{N}] ; F_{\sim}-$ вынуждающая сила $[\mathrm{N}]$.

\section{1. Возбуждение вынужденных колебаний}

Создание вынуждающей силы реализовано в установке за счет магнитной индукции (рис. 2). Согласно закону 


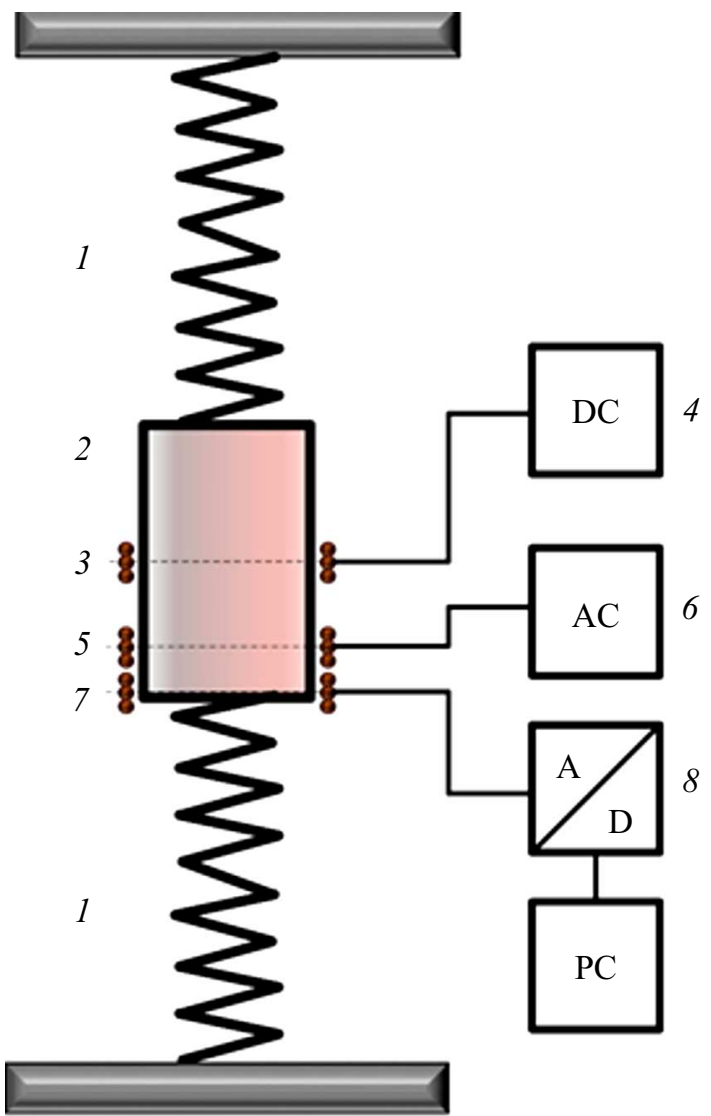

Рис. 1. Схема экспериментальной установки: 1 - пружина, 2 - магнит, 3, 5, 7 - катушки, 4 - источник постоянного тока, 6 - генератор переменного тока, 8 - цифровая лаборатория.

Ампера, на катушку 5 действует сила

$$
F_{A_{\sim}}=\oint_{\sigma} N I_{\sim}\left(\mathbf{d} \mathbf{l} \times \mathbf{B}_{\mathbf{D}}\right),
$$

где $I_{\sim}$ - мгновенная амплитуда переменного тока в катушке 5 (рис. 1); $N$ - число витков; dl - элемент обхода контура катушки.

В качестве модели поля постоянного магнита 2 (рис. 1,2$) \mathbf{B}(x, y, z)$ примем магнитное поле магнитного диполя $\mathbf{B}_{\mathbf{D}}[5,6]$. В цилиндрической системе координат (рис. 2) $x$-компонента и радиальная компонента вектоpa $\mathbf{B}_{\mathbf{D}}$ :

$$
\begin{gathered}
B_{x}=\frac{\mu_{0} m}{4 \pi\left(x^{2}+\rho^{2}\right)^{5 / 2}}\left(2 x^{2}-\rho^{2}\right), \\
B_{\rho}=\frac{\mu_{0}}{4 \pi} \frac{3 m x \rho}{\left(x^{2}+\rho^{2}\right)^{5 / 2}},
\end{gathered}
$$

где $\rho$ - радиус витка $[\mathrm{m}] ; \mu_{0}=1.25 \cdot 10^{-6}$ - магнитная постоянная $\left[\mathrm{N} / \mathrm{A}^{2}\right], m-$ магнитный дипольный момент груза-магнита $\left[\mathrm{A} \cdot \mathrm{m}^{2}\right] ; x-$ смещение центра магнитагруза относительно центра катушки $[\mathrm{m}]$.
Согласно третьему закону Ньютона, на магнит будет действовать сила $F_{A_{\sim}}$. Компонента этой силы в направлении оси $x$ определяется выражением

$$
F_{\sim x}=N I_{\sim}(2 \pi \rho) B_{\rho}=N I_{\max }(2 \pi \rho) B_{\rho} \cos \omega t,
$$

где $I_{\max }$ - амплитуда переменного тока, $[\mathrm{A}], \omega-$ циклическая частота переменного тока, [1/s].

\section{2. Возбуждение нелинейных колебаний}

Нелинейные колебания возбуждаются постоянным током в катушке 3 (рис. 1,2). С учетом сказанного выше сила, действующая со стороны катушки 3 на магнит 2, может быть представлена в виде

$$
\begin{aligned}
F_{\mathrm{M}} & =N I_{0}(2 \pi \rho) B_{\rho}=\left(\frac{3 \mu_{0} N I_{0} m \rho^{2}}{2}\right)\left(\frac{x}{\left(x^{2}+\rho^{2}\right)^{5 / 2}}\right) \\
& \approx\left(\frac{3 \mu_{0} N I_{0} m \rho^{2}}{2}\right)\left(\frac{x}{\rho}\right)\left[1-\frac{5}{2}\left(\frac{x}{\rho}\right)^{2}\right]=q_{1} x-q_{2} x^{3}
\end{aligned}
$$

где $q_{1}=3 \mu_{0} N m \rho I_{0} / 2, q_{2}=5 \mu_{0} N m I_{0} / 4 \rho, I_{0}$ - величина постоянного тока, протекающего через катушку 3.

В приведенной выше формуле мы использовали первые два члена бинома Ньютона для разложения функции $\left[1+\left(\frac{x}{\rho}\right)^{2}\right]^{-\frac{5}{2}}$ по степеням $\left(\frac{x}{\rho}\right)^{2}$, предполагая малые смещения магнита по отношению к центральному витку катушки 3. В этом случае принимается, что $x$ координата магнита удовлетворяет условию $x(t) \ll \rho$.

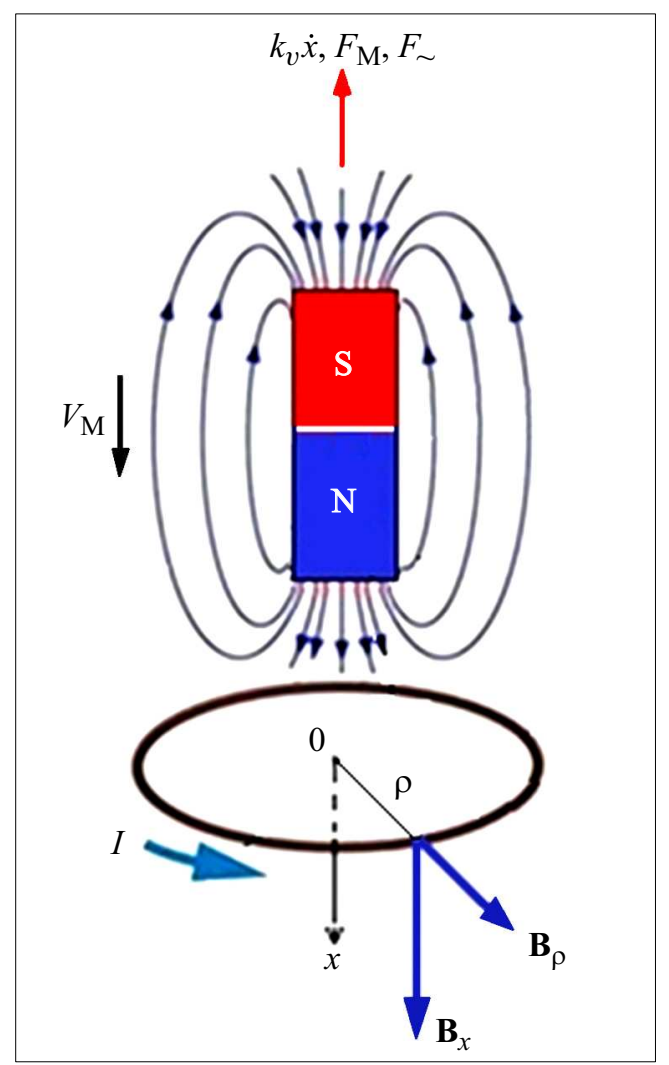

Pис. 2. Схема движения магнита относительно кольца катушки. 


\section{3. Регистрация колебаний}

При движении магнита 2 относительно катушки 7 в ней индуцируется ЭДС:

$$
\varepsilon=-\int_{\mathbf{S}} \frac{\partial \mathbf{B}}{\partial t} d \mathbf{S}+\oint_{\sigma}(\mathbf{v} \times \mathbf{B}) \mathbf{d} \mathbf{l},
$$

где $\mathbf{v}$ - вектор скорости элемента проводника $[\mathrm{m} / \mathrm{s}]$.

В выражении (2) первое слагаемое обусловлено изменением индукции магнитного поля в точке пространства, а второе определяется магнитной силой. Поскольку в установке подвижным является магнит, ЭДС в катушке определяется первым слагаемым в выражении $(2)[5,6]$

$$
\varepsilon=\frac{3 \mu_{0} m \rho^{2} x}{2\left(\rho^{2}+x_{m}^{2}\right)^{\frac{5}{2}}} v_{m},
$$

где $v_{m}=d x / d t-$ скорость центра магнита $[\mathrm{m} / \mathrm{s}]$.

Принимается, что магнит совершает гармонические колебания $x=A_{m} \cos (\omega t)$, это позволяет считать, что амплитуда ЭДС пропорциональна амплитуде колебаний магнита $A_{m}$.

Катушка 7 выполняет регистрацию колебаний и одновременно создает диссипативную силу. В природе всегда существуют силы, препятствующие механическому движению. В частности - силы трения между твердыми телами и вязкими жидкостями. Например, можно рассмотреть затухающие колебания пружинного маятника, погруженного в жидкость. Для анализа и учета сил трения необходимо выбрать наиболее адекватную эксперименту математическую модель. В частности, силу, приводящую к затуханию движения маятника, погруженного в жидкость, можно считать пропорциональной скорости движения тела. В дальнейшем будем считать, что сила торможения пропорциональна скорости и направлена в противоположную сторону:

$$
F_{f r}=-k_{v} \dot{x} .
$$

Сила торможения реализована в установке за счет электромагнитной индукции: груз представляет собой постоянный магнит, движущийся относительно катушек индуктивности. Коэффициент сопротивления (диссипации) можно определить по формуле [5]

$$
k_{v}=\frac{N^{2}(2 \pi \rho)^{2} B_{\rho}^{2}}{R+r_{0}},
$$

где $R, r_{0}$ - соответственно сопротивление резистора и внутреннее сопротивление катушки.

Запишем уравнение движения (1) в виде

$$
\ddot{x}+2 \beta \dot{x}+\omega_{0}^{2} x=f_{1} x-\gamma x^{3}+f_{0} \cos \omega t,
$$

где $f_{1}=q_{1} / M, \quad \gamma=q_{2} / M, \quad f_{0}=N I_{\max }(2 \pi \rho) B_{\rho} / M$; $\beta=k_{v} / 2 M-$ коэффициент затухания [1/s]; $\omega_{0}^{2}=\left(k_{1}+k_{2}\right) / M-$ квадрат циклической частоты свободных гармонических колебаний $\left[1 / \mathrm{s}^{2}\right]$; или

$$
\ddot{x}+2 \beta \dot{x}+\omega_{1}^{2} x=-\gamma x^{3}+f_{0} \cos \omega t,
$$

где

$$
\omega_{1}=\omega_{0} \sqrt{1-q I_{0}}, \quad q=3 \mu_{0} N m \rho / 2 M \omega_{0}^{2} .
$$

Осциллятор Дуффинга (5) или осциллятор с кубической нелинейностью, как в нашем примере, является распространенной моделью в теории нелинейных колебаний $[1,2]$.

\section{4. Уравнение свободных колебаний с малой амплитудой}

Свободные колебания с малой амплитудой (пренебрегаем в (5) слагаемым $-\gamma x^{3}$ и вынуждающей силой $\left.f_{0}=0\right)$ описываются уравнением:

$$
\ddot{x}+2 \beta \dot{x}+\omega_{1}^{2} x=0 .
$$

В этом случае, согласно (6), происходит сдвиг частоты свободных колебаний. При $I_{0}>0$ сдвиг $\omega_{1}-\omega_{0}<0$, а при $I_{0}<0$ сдвиг $\omega_{1}-\omega_{0}>0$.

\section{5. Свободные колебания с большой амплитудой}

Свободные колебания с учетом слагаемого $-\gamma x^{3}$ и отсутствием вынуждающей силы $f_{0}=0$ описываются уравнением

$$
\ddot{x}+2 \beta \dot{x}+\omega_{1}^{2} x=-\gamma x^{3} .
$$

Решение ищем в виде

$$
x=A \cos \omega t,
$$

тогда

$$
x^{3}=A^{3}\left(\frac{3}{4} \cos \omega t+\frac{1}{4} \cos 3 \omega t\right) .
$$

Таким образом, в спектре силы, приложенной к магниту со стороны катушки 3, присутствуют первая и третья гармоники. Как показано в работе [1], амплитуда колебаний на частоте $3 \omega\left(\varepsilon A^{3} / 32, \varepsilon \ll 1\right)$ существенно меньше амплитуды колебаний на частоте $\omega(A)$, и такие колебания не регистрируется нашей аппаратурой. Пренебрегаем третьей гармоникой (ангармоничностью) в (7), тогда частота нелинейных свободных колебаний

$$
\begin{gathered}
\omega_{2}=\omega_{0} \sqrt{1-q I_{0}+\left(\frac{3 \gamma}{4}\right) A^{2}}=\omega_{1} \sqrt{1+\left[\frac{3 \gamma}{4\left(1-q I_{0}\right)}\right] A^{2}} \\
\approx \omega_{1}+\delta A^{2},
\end{gathered}
$$

где $\delta=3 \gamma / 8 \omega_{1}$.

Поскольку собственная частота $\omega_{2}(8)$ зависит от амплитуды колебаний, нелинейность носит „реактивный“характер. Мы рассмотрели случай $\gamma>0$ и $q I_{0}<1$, реализуемый в нашем эксперименте. В этом случае в фазовом пространстве существует одна точка равновесия $\left(\omega_{1}\right)$. При $q I_{0}>1$ (случай, не реализуемый в нашем эксперименте) в фазовом пространстве существуют три 
Значения параметров при расчете резонансных кривых (рис. 3)

\begin{tabular}{c|c|c|c|c|c}
\hline № кривой & $\beta, 1 / \mathrm{s}$ & $\gamma, 1 / \mathrm{s}^{2} \mathrm{~m}^{2}$ & $f_{0}, \mathrm{~m} / \mathrm{s}^{2}$ & $f_{1}, 1 / \mathrm{s}^{2}$ & $v_{0}=\omega_{0} / 2 \pi, \mathrm{Hz}$ \\
\hline 1 & 0.14 & 117000 & 0.27 & 280 & 6.31 \\
2 & 0.14 & 117000 & 0.17 & 280 & 6.31 \\
3 & 0.14 & 117000 & 0.06 & 280 & 6.31 \\
4 & 0.09 & 0 & 0.11 & 280 & 6.31 \\
5 & 0.14 & 117000 & 0.27 & 280 & 6.31 \\
6 & 0.14 & 117000 & 0.17 & 280 & 6.31 \\
7 & 0.14 & 117000 & 0.06 & 280 & 6.31
\end{tabular}

точки равновесия, две из которых являются неустойчивыми. В этом случае колебания носят хаотический характер.

Зависимость частоты собственных колебаний $\omega_{2}$ от амплитуды (8) отражает их неизохронность, что является одним из типичных нелинейных явлений, наряду с ангармоничностью и мультистабильностью.

\section{6. Вынужденные колебания и резонанс}

В уравнении (5) пренебрегаем $x^{3}$, но учитываем вынуждающую силу. Решение уравнения (5) в этом случае хорошо известно $[1,2]$ :

$$
x=A_{0} \cos (\omega t+\varphi),
$$

где

$$
\begin{gathered}
A_{0}=\frac{f_{0}}{\left[\left(\omega_{1}^{2}-\omega^{2}\right)^{2}+4 \omega^{2} \beta^{2}\right]^{1 / 2}}, \\
\tan \varphi=\frac{2 \beta \omega}{\omega_{1}^{2}-\omega^{2}} .
\end{gathered}
$$

При больших амплитудах $A_{0}$, учитывая $x^{3}$, решение уравнения (5) ищется, например, методом медленно меняющихся амплитуд (MМА) [1,2] и может быть представлено в виде:

$$
\omega=\omega_{1}+\delta A_{0}^{2} \mp \sqrt{\left(\frac{f_{0}}{2 A_{0} \omega_{1}}\right)^{2}-\beta^{2}} .
$$

Уравнение $\omega=\omega_{1}+\delta A_{0}^{2}$ представляет собой параболу, которая является ,Позвоночником“ нелинейных резонансов и представляет локус пиков кривых резонансов. При $I_{0}>0$ локус пиков нелинейных резонансов направлен в сторону больших от $\omega_{1}$ частот, а при $I_{0}<0$ в сторону меньших от $\omega_{1}$ частот.

На рис. 3 представлены теоретические графики (1-3) зависимости амплитуды нелинейных колебаний от частоты при различных значениях амплитуды вынуждающей силы и $I_{0}>0$, построенные с использованием выражения (10). Аналогичные графики (5-7) показаны при $I_{0}<0$. Кривая 4 соответствует линейным вынужденным колебаниям и получена с использованием выражения (9). Кривые 8 („позвоночник“) построены

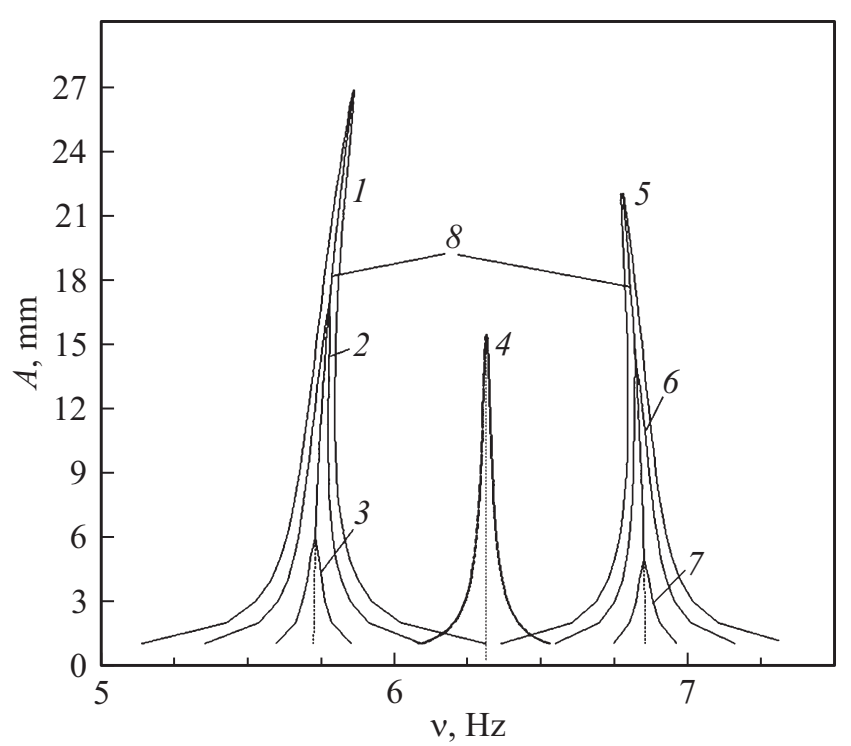

Рис. 3. Теоретические графики резонансных кривых зависимости амплитуды колебаний $A$ от частоты $v$ для нелинейных вынужденных колебаний $1-3\left(I_{0}>0\right)$ и 5-7 $\left(I_{0}<0\right)$, а также линейных вынужденных колебаний 4 .

по формуле (8). В таблице представлены значения задающих параметров колебаний.

Разработана численная модель нелинейных колебаний, основанная на уравнении (4). Решение базировалось на методе Эйлера-Кромера. Модель позволяет изучать динамику нелинейных колебаний в процессе установления стационарного состояния. На рис. 4 показаны „разгонные“ кривые при параметрах колебаний, указанных в таблице, и частоте вынуждающих колебаний $5.73 \mathrm{~Hz}$. На рис. 4 (верхняя часть) точки 1-3 соответствуют установишимся колебаниям с амплитудами $A_{1}, A_{2}, A_{3}$ (нижняя часть).

\section{3. Эксперимент}

Эксперимент (рис. 1) выполнялся при следующих параметрах колебаний пружинного маятника: стальные пружины 1 имели коэффициент упругости $k=27.5 \mathrm{~N} / \mathrm{m}$; цилиндрический магнит 2 длиной $0.02 \mathrm{~m}$, диаметром $0.016 \mathrm{~m}$, массой $0.035 \mathrm{~kg}$, с магнитным дипольным моментом $m=1.4 \mathrm{~A} \cdot \mathrm{m}^{2}$; индукционные катушки: 3 (число витков $N=120) ; 4(N=120)$ и $5(N=80)$ с внутренним диаметром $0.02 \mathrm{~m}$ имеют высоту $0.008 \mathrm{~m}$. Центральный виток катушки 3 совмещен с центром магнита 2 в положении равновесия; центральный виток катушки 4 смещен относительно центра магнита на 0.014 m; центр катушки 5 смещен относительно центра магнита на 0.024 m. Катушка 3 подключена к источнику постоянного напряжения HY1503D, а катушка 4 - к источнику переменного напряжения АКИП-3409/3. Постоянный ток в катушке 3 изменялся в диапазоне от -0.5 до $+0.5 \mathrm{~A}$. Амплитуда переменного тока изменялась в диапазоне 

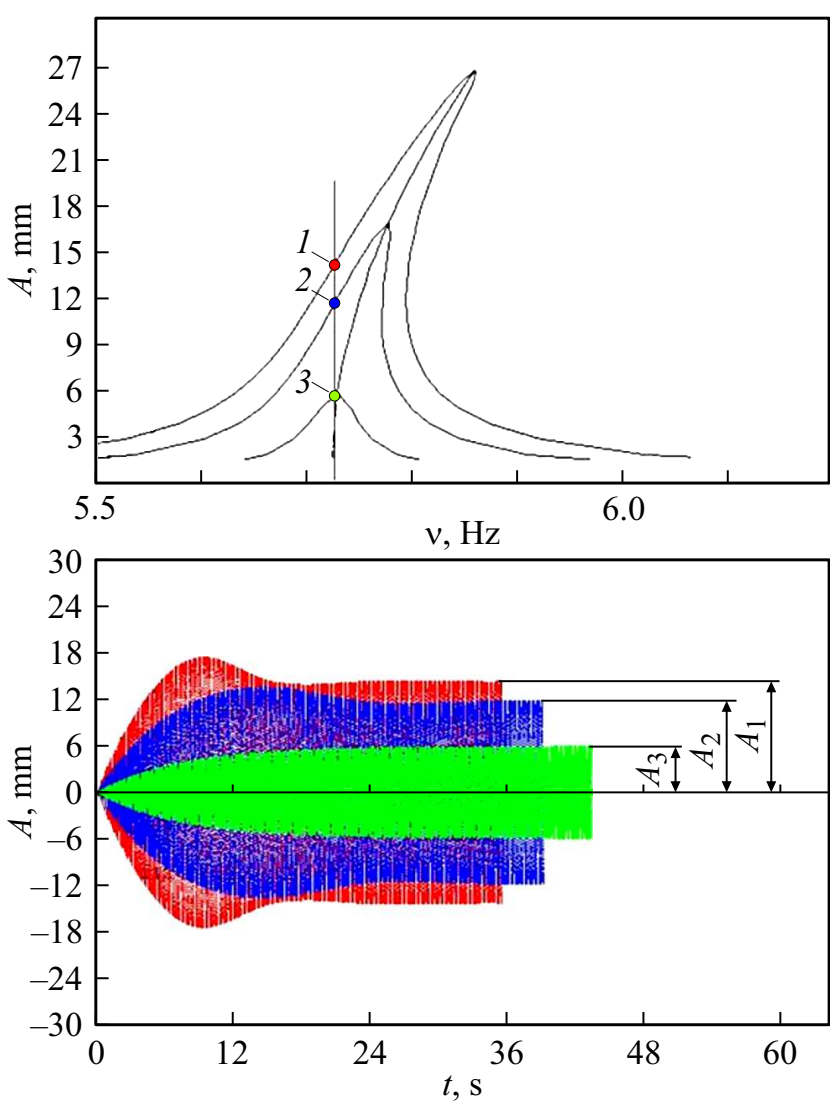

Рис. 4. Графики нелинейных колебаний, полученные с использованием приближенного решения (10) - верхняя часть рисунка и „разгонные“ кривые зависимости амплитуды $A$ от времени $t$, полученные численным моделированием - нижняя часть рисунка.

от 0 до $0.1 \mathrm{~A}$, а частота переменного тока изменялась в диапазоне от 4 до $8 \mathrm{~Hz}$. Катушка 5, выходные клеммы которой замкнуты с помощью резистора, имеющего сопротивление $R=2 \Omega$, соединена с цифровой лабораторией, регистрирующей ЭДС катушки в режиме осциллографа. Осциллограф использовался с частотой выборки $10^{3} 1 / \mathrm{s}$. Результаты измерений и расчетов представлены на рис. 5 .

\section{1. Сравнение результатов расчета и экспериментальных данных}

Чтобы проверить приближенную аналитическую модель (10), мы выполнили ряд экспериментов, демонстрирующих нелинейный характер колебаний предложенного пружинного осциллятора. Предварительно мы выполнили ряд экспериментов, позволяющих откалибровать и измерить соответствующие параметры колебательной системы.

1. Мы измерили частоту свободных колебаний пружинного маятника $\omega_{0}$ (генераторы постоянного и переменного тока отключены) и собственную частоту $\omega_{1}$ при различных величинах постоянного тока $I_{0}$ (через

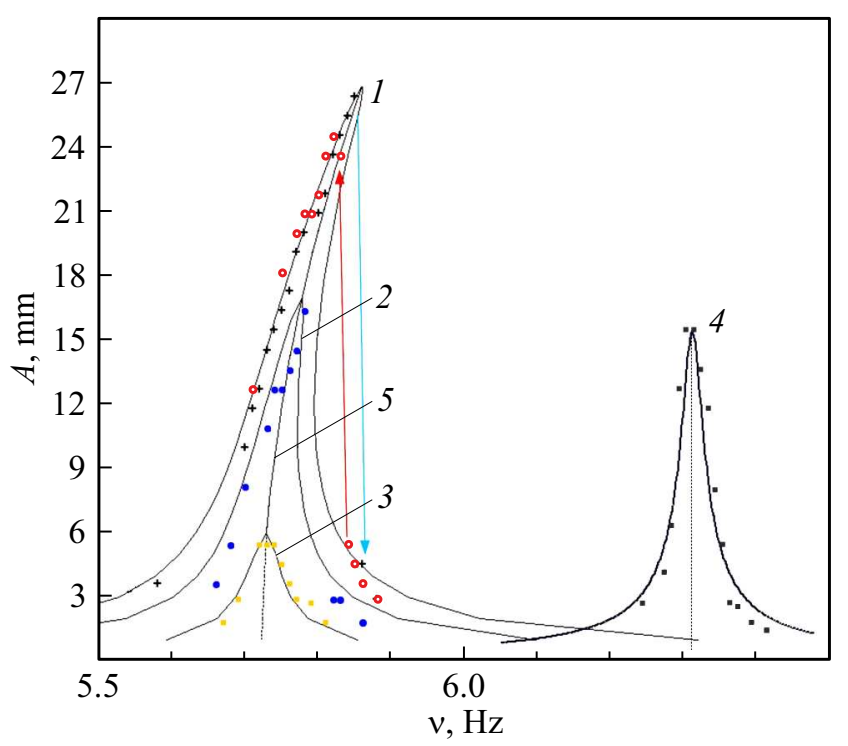

Рис. 5. Экспериментальные результаты и теоретические графики резонансных кривых зависимости амплитуды колебаний $A$ от частоты $v$ для нелинейных вынужденных колебаний $1-3\left(I_{0}=+0.4 \mathrm{~A}\right)$, а также линейных вынужденных колебаний 4. Черные кресты (частота переменного тока растет) и полые кружки (частота переменного тока убывает) соответствуют амплитуде переменного тока $+0.08 \mathrm{~A}$, стрелки показывают гистерезис. Черные кружки и квадраты соответствуют амплитудам переменного тока +0.04 и $0.02 \mathrm{~A}$.

катушку 3) и отключенном генераторе переменного тока (через катушку 5). Эксперименты с хорошей степенью точности подтвердили теоретическую формулу (6) и позволили подобрать параметр $q$.

2. В наших экспериментах реализуется условие $q I_{0}<1$, что позволило получить ряд типичных нелинейных явлений, указанных в теоретическом разделе.

3. Мы выполнили предварительный эксперимент при выключенном генераторе постоянного тока и изменяли частоту переменного тока в диапазоне, близком к частоте $\omega_{0}=6.31 \mathrm{~Hz}$. В этом случае наблюдался хорошо изученный линейный резонанс вынужденных колебаний пружинного маятника, описываемый формулой (9). Результаты эксперимента и сравнение с теорией приведены на рис. 5 .

4. Был выполнен эксперимент, определяющий зависимость амплитуды ЭДС от амплитуды колебаний магнита. Линейная зависимость, предсказанная формулой (3), выполнялась в диапазоне используемых в эксперименте параметров.

\section{2. Нелинейный резонанс}

Нелинейный резонанс регистрировался при значении постоянного тока $I_{0}=+0.4 \mathrm{~A}$. Частота переменного тока изменялась в диапазоне от 4 до $8 \mathrm{~Hz}$ при следующих значениях амплитуды переменного тока: $+0.02 \mathrm{~A}$, +0.04 А и +0.08 А. При этом амплитуда вынуждающей 
силы, приложенной к магниту со стороны катушки 4, равнялась 9.5, 6.0, $2.1 \mathrm{mN}$. Коэффициент затухания $\beta=0.141 / \mathrm{s}$. На этом же рисунке приведены теоретические кривые, представленные формулой (10). При получении экспериментальных точек (черные кресты и полые кружки на рис. 5), соответствующих амплитуде переменного тока $+0.08 \mathrm{~A}$, частота переменного тока, протекающего через катушку 5, увеличивалась от 5.5 до $6 \mathrm{~Hz}$ (черные кресты), а затем последовательно уменьшалась (полые кружки). При этом эксперимент демонстрирует скачкообразное уменьшение (стрелка вниз), или увеличение (стрелка вверх) амплитуды колебаний при частоте $\sim 5.85 \mathrm{~Hz}$. Наблюдается гистерезис, обозначенный на рисунке стрелками. В области гистерезиса колебательная система является бистабильной. Менее выраженный „скачок“ амплитуды колебаний наблюдается при частоте $\sim 5.78 \mathrm{~Hz}$ (черные кружки, соответствующие амплитуде переменного тока +0.04 A).

Экспериментальные результаты, приведенные на рис. 5, также демонстрируют зависимость частоты нелинейных колебаний маятника от амплитуды, что отражает их неизохронность.

Получено хорошее согласие приближенной теоретической модели (10), численного решения уравнения (4) и экспериментальных данных.

\section{Заключение}

Динамика линейных колебаний обычно используется как первое приближение реальной физической системы. Мы провели исследование более реального физического устройства, позволяющего исследовать нелинейные колебания и резонанс пружинного маятника. Отличительной особенностью предложенной установки является унификация возбуждения нелинейных колебаний, их регистрация и создание диссипативных сил только за счет электромагнитной индукции. Предложена теоретическая модель, приводящая к уравнению Дуффинга, аналитическое и численное решения, а также приведены экспериментальные результаты, хорошо согласующиеся с теорией. Экспериментальные результаты демонстрируют зависимость частоты нелинейных колебаний маятника от амплитуды, что отражает их неизохронность. Пики кривых нелинейных резонансов расположены на параболе, которая является „позвоночником“ этих резонансов. При этом эксперимент демонстрирует скачкообразное уменьшение (или увеличение) амплитуды колебаний при частоте $\sim 5.85 \mathrm{~Hz}$. Наблюдается гистерезис, в области которого колебательная система является бистабильной.

\section{Список литературы}

[1] Кузнецов А.П., Кузнецов С.П., Рыскин Н.М. Лекции по теории колебаний и волн. Нелинейные колебания. Саратов: СГУ им. Н.Г. Чернышевского, 2011. 314 с.

[2] Паршаков А.Н. Физика колебаний. Пермь: Пермский гос. тех. ун-т, 2010. 302 c.

[3] Kraftmakher Y. // Eur. J. Phys. 2007. Vol. 28. P. 1007-1020. DOI: $10.1088 / 0143-0807 / 28 / 5 / 023$

[4] Бутиков Е.И. // Компьютерные инструменты в образовании. 2008. № 1. С. 30-42.

[5] Зейликович И.С., Никитин А.В., Матеикий Н.В., Василевич А.Е., Дягель Д.Ю., Заман В.А., Шункеев К.Ш., Бекешев А.З. // Физическое образование в вузах. 2018. Т. 24. № 4. C. $122-136$.

[6] Carpena P. // Am. J. Phys. 1997. Vol. 65. N 2. P. 135-140.

\section{Конфликт интересов}

Авторы заявляют, что у них нет конфликта интересов. 